\title{
Improved irritative voiding symptoms 3 years after stereotactic body radiation therapy for prostate cancer
}

\section{Zaker Rana ${ }^{1+}$, Robyn A. Cyr ${ }^{1+}$, Leonard N. Chen ${ }^{1}$, Brian S. Kim ${ }^{1}$, Rudy A. Moures ${ }^{1}$, Thomas M. Yung $^{1}$, Siyuan Lei ${ }^{1}$, Brian T. Collins ${ }^{1}$, Simeng Suy ${ }^{1}$, Anatoly Dritschilo ${ }^{1}$, John H. Lynch ${ }^{2}$ and Sean P. Collins ${ }^{1}{ }^{*}$}

${ }^{1}$ Department of Radiation Medicine, Georgetown University Hospital, Washington, DC, USA

${ }^{2}$ Department of Urology, Georgetown University Hospital, Washington, DC, USA

\section{Edited by:}

Alan Jay Katz, Flushing Radiation Oncology Services, USA

Reviewed by:

Rachelle Lanciano, Delaware County Memorial Hospital, USA

Josephine Kang, Flushing Radiation

Oncology Services, USA

*Correspondence:

Sean P. Collins, Department of

Radiation Medicine, Georgetown University Medical Center, 3800

Reservoir Road Northwest,

Washington, DC 20007, USA

e-mail: spc9@georgetown.edu

${ }^{\dagger}$ Zaker Rana and Robyn A. Cyr have contributed equally to this work.
Background: Irritative voiding symptoms are common in elderly men and following prostate radiotherapy. There is limited clinical data on the impact of hypofractionated treatment on irritative voiding symptoms. This study sought to evaluate urgency, frequency, and nocturia following stereotactic body radiation therapy (SBRT) for prostate cancer.

Methods: Patients treated with SBRT monotherapy for localized prostate cancer from August 2007 to July 2011 at Georgetown University Hospital were included in this study. Treatment was delivered using the CyberKnife ${ }^{\circledR}$ with doses of $35-36.25 \mathrm{~Gy}$ in five fractions. Patient-reported urinary symptoms were assessed using the International Prostate Symptom Score (IPSS) before treatment and at 1, 3, 6, 9, and 12 months post-treatment and every 6 months thereafter.

Results: Two hundred four patients at a median age of 69 years received SBRT with a median follow-up of 4.8 years. Prior to treatment, $50.0 \%$ of patients reported moderate to severe lower urinary tract symptoms (LUTS) and $17.7 \%$ felt that urinary frequency was a moderate to big problem. The mean prostate volume was $39 \mathrm{cc}$ and $8 \%$ had prior procedures for benign prostatic hyperplasia. A mean baseline IPSS-irritative (IPSS-I) score of 4.8 significantly increased to 6.5 at 1 month $(p<0.0001)$, however returned to baseline at 3 months $(p=0.73)$. The IPSS-I score returned to baseline in $91 \%$ of patients by 6 months and $96 \%$ of patients by 2 years. Transient increases in irritative voiding symptoms were common at 1 year. The mean baseline IPSS-I score decreased to 4.4 at 24 months $(p=0.03)$ and 3.7 at 36 months $(p<0.0001)$. In men with moderate to severe LUTS (IPSS $\geq 8$ ) at baseline, the mean IPSS-I decreased from a baseline score of 6.8-4.9 at 3 years post-SBRT. This decrease was both statistically $(p<0.0001)$ and clinically significant (minimally important difference $=1.45$ ). Only $14.6 \%$ of patients felt that urinary frequency was a moderate to big problem at 3 years post-SBRT ( $p=0.23$ ).

Conclusion: Treatment of prostate cancer with SBRT resulted in an acute increase in irritative urinary symptoms that peaked within the first month post-treatment. Irritative voiding symptoms returned to baseline in the majority of patients by 3 months post-SBRT and were actually improved from baseline at 3 years post-SBRT.

Keywords: prostate cancer, SBRT, CyberKnife, IPSS, irritative, overactive bladder

\section{BACKGROUND}

Irritative voiding symptoms are a common problem of male aging (1). In men $>75$ years old, the prevalence of these symptoms may be as high as $40 \%$ (2). Comorbidities may increase the risk

Abbreviations: ADT, androgen deprivation therapy; BED, biologically effective dose; $\mathrm{BPH}$, benign prostatic hyperplasia; $\mathrm{CT}$, computed tomography; CTV, clinical target volume; DVH, dose-volume histogram; EPIC, expanded prostate index composite; EQD2, equivalent dose in 2-Gy fractions; GTV, gross target volume; Gy, gray; IMRT, intensity modulated radiation therapy; IPSS, international prostate symptom score; IRB, institutional review board; LUTS, lower urinary tract symptoms; MID, minimally important difference; $\mathrm{MR}$, magnetic resonance; PTV, planning target volume; QoL, quality of life; SD, standard deviation; SBRT, stereotactic body radiation therapy. of irritative voiding symptoms (3). They commonly develop or worsen following prostate external beam radiation therapy (EBRT) and may adversely affect a patient's quality of life $(4,5)$. Patients report the development of urinary urgency, urinary frequency, and nocturia days to weeks after the start of treatment and generally resolve weeks to months following completion of EBRT. Treatment related factors such as utilization of brachytherapy (6) may impact the risk of irritative voiding symptoms. Anti-cholinergic medications may decrease these symptoms (6), but are commonly discontinued due to associated dry mouth and constipation (7).

Urinary urgency is defined as the complaint of a sudden compelling desire to pass urine, which is difficult to defer (8). Urgency may promote urinary incontinence in patients with pelvic floor 
muscle weakness and/or poor mobility. Urinary frequency and nocturia may increase by reducing voiding intervals (9). Nocturia is defined as a self-report of two or more voiding episodes nightly $(10,11)$. It causes sleep loss, daytime fatigue, and depression, which adversely affects an individual patient's quality of life (12). In the elderly, nocturia may even increase the incidence of falls $(13,14)$.

Stereotactic Body Radiation Therapy (SBRT) is a safe and effective treatment for clinically localized prostate cancer (15-19). The larger dose per fraction utilized in SBRT offers the potential radiobiological benefits of hypofractionation (20). The low PSA nadirs obtained with SBRT (21) suggest it is an ablative procedure, which eradicates both cancerous and normal epithelium. The size of the prostate decreases by $35 \%$ within the first 2 years following the completion of SBRT (22). Initial reports suggest that the incidence of acute irritative voiding symptoms following SBRT is comparable to other external radiotherapy modalities, and may be less than brachytherapy (15-17). The goal of this study is to report the incidence and prevalence of irritative voiding symptoms following SBRT for clinically localized prostate cancer.

\section{MATERIALS AND METHODS PATIENT SELECTION}

Georgetown University Hospital established its Prostate SBRT Program in 2006. As of June 2014, 750 prostate cancer patients have been treated with SBRT. At the inception of the program, a prospective database was established to record baseline patient characteristics. At each follow-up visit, toxicity and quality of life data have also been prospectively collected and recorded. Patients eligible for this study had SBRT without supplemental conventional radiation therapy for clinically localized prostate cancer and a minimum of 3 years of follow-up. Internal Review Board (IRB) approval was obtained for retrospective review of the database.

\section{SBRT TREATMENT PLANNING AND DELIVERY}

Stereotactic body radiation therapy treatment planning and delivery were conducted as previously described $(23,24)$. Briefly, four to six stranded gold fiducials (1013-2-2, Best Medical International, Inc., Springfield, VA, USA) were placed into the prostate with two to three needle applicators via a transrectal or transperineal approach. Fused computed tomography (CT) and magnetic resonance $(\mathrm{MR})$ images were used for treatment planning. The clinical target volume (CTV) included the prostate and the proximal seminal vesicles. The planning target volume (PTV) equaled the CTV expanded $3 \mathrm{~mm}$ posteriorly and $5 \mathrm{~mm}$ in all other dimensions. The prescription dose was 35-36.25 Gy to the PTV delivered in five fractions of $7-7.25$ Gy over $1-2$ weeks. The prescription isodose line was limited to $\geq 75 \%$, which limited the maximum prostatic urethra dose to $133 \%$ of the prescription dose. Bladder volume receiving $37 \mathrm{~Gy}$ was limited to $<5 \mathrm{cc}$. The bladder dose-volume histogram (DVH) goals were for $<40 \%$ of the bladder volume to receive $50 \%$ of the prescribed dose and $<10 \%$ to receive $100 \%$ of the dose. The membranous urethra was contoured and evaluated with DVH analysis during treatment planning using Multiplan (Accuray Inc., Sunnyvale, CA, USA). The DVH goal was for $<50 \%$ of the membranous urethra to receive $37 \mathrm{~Gy}$. To minimize the risk of local recurrence, the dose to the prostatic urethra was not constrained (25). Prostate position was verified during treatment using paired, orthogonal x-ray images (26).

\section{FOLLOW-UP AND STATISTICAL ANALYSIS}

Prospective quality of life data was obtained on the first day of SBRT treatment and during routine follow-up visits every 3 months for the first year and every 6 months for the second and third years. Patient-reported irritative voiding symptoms were assessed via the International Prostate Symptom Score (IPSS), a validated questionnaire where higher scores indicate more severe symptoms (27). The IPSS includes three questions related to irritative voiding symptoms (frequency, urgency, and nocturia). For the frequency and urgency questions, the responses were grouped into four clinically relevant categories (never, less than half the time, half or more than half the time, and almost always). For the nocturia question, the responses were grouped into four clinically relevant categories (none, 1 time, 2 times, and $\geq 3$ times).

Table 1 | Baseline patient and treatment characteristics.

\begin{tabular}{|c|c|c|}
\hline & & $\begin{array}{l}\text { Patients } \\
(N=204)(\%\end{array}$ \\
\hline \multirow[t]{4}{*}{ Age (y/o) } & Median 69 (48 90) & \\
\hline & Age $\leq 60$ & 12.7 \\
\hline & $60<$ Age $\leq 70$ & 46.6 \\
\hline & Age $>70$ & 40.7 \\
\hline \multirow[t]{3}{*}{ Race } & White & 54.4 \\
\hline & Black & 38.7 \\
\hline & Other & 7.8 \\
\hline \multirow[t]{3}{*}{ Charlson comorbidity index } & $\mathrm{CCl}=0$ & 65.2 \\
\hline & $\mathrm{CCl}=1$ & 21.1 \\
\hline & $\mathrm{CCl} \geq 2$ & 13.7 \\
\hline \multirow[t]{2}{*}{ Body mass index (BMI) } & Median 27.60 (15.02-44.96) & \\
\hline & $\mathrm{BMI} \geq 30$ & 30.5 \\
\hline \multirow[t]{2}{*}{ Partner status } & Married or partnered & 76.0 \\
\hline & Not partnered & 24.0 \\
\hline \multirow[t]{2}{*}{ Employment status } & Working & 48.0 \\
\hline & Not working & 52.0 \\
\hline Median prostate volume (cc) & Median 39 (11.6-138.7) cc & \\
\hline Procedure for $\mathrm{BPH}$ & & 7.8 \\
\hline$\alpha_{1 A}$ Inhibitor usage & & 27.9 \\
\hline \multirow[t]{3}{*}{ Risk groups (D'Amico's) } & Low & 39.7 \\
\hline & Intermediate & 52.0 \\
\hline & High & 8.3 \\
\hline ADT & & 14.2 \\
\hline \multirow[t]{2}{*}{ SBRT dose } & $36.25 \mathrm{~Gy}$ & 87.7 \\
\hline & $35 \mathrm{~Gy}$ & 12.3 \\
\hline \multirow[t]{4}{*}{ Baseline IPSS score } & Median $=7.5(0-33)$ & \\
\hline & Mild (0-7) & 50.0 \\
\hline & Moderate (8-19) & 43.6 \\
\hline & Severe $(\geq 20)$ & 6.4 \\
\hline
\end{tabular}


As previously reported, nocturia was defined as urinating two or more times per night (2). The IPSS-irritative (IPSS-I) subscore has been previously defined as the sum of the scores for questions 2, 4, and 7 (28). Overall IPSS-I scores range from 0 to 15. IPSS-I resolution was defined as a return to within one point of the baseline score (29). Bother with urinary frequency was assessed via Question 4e of the Expanded Prostate Index Composite (EPIC)26 (30), for which responses were grouped into three clinically relevant categories (no problem, small problem, and moderate to big problem).

Wilcoxon signed-rank test and Student $t$-test were used to assess the differences in ongoing toxicity and quality of life scores in comparison to baseline. To limit the effect of attrition bias, statistical analysis was limited to time points in which $\geq 80 \%$ of patient data were available. Sample medians and ranges were used to describe continuous variables. Actuarial likelihood estimates for time to IPSS-I resolution were determined using the Kaplan-Meier method. To statistically compare changes between time points, the levels of responses were assigned a score and the significance of the mean changes in the scores was assessed by the Wilcoxon signed-rank test. Binary logistic regression was used in the multivariate analysis to search for possible predicting factors for IPSS-I improvement. The endpoint for this analysis was an IPSS-I score at least one point lower than baseline at 3 years post-SBRT. Baseline characteristics including age, race, Charlson Comorbidity Index (CCI), risk group, partner status, work status, prostate volume, baseline $\alpha_{1 \mathrm{~A}}$ inhibitor use, baseline androgen deprivation therapy (ADT) use, previous history of transurethral resection of the prostate (TURP), and treatment dose were included as variables in the logistic regression model. The minimally important difference (MID) in IPSS-I score was defined as a change of one-half standard deviation (SD) from the baseline (31). A non-paired Student $t$-test was used to determine if the magnitude of changes in the IPSS-I score was significantly different between men with baseline mild lower urinary tract symptoms (LUTS) (IPSS $<8$ ) and moderate to severe LUTS (IPSS $\geq 8$ ) (32).

\section{RESULTS}

From February 2008 to July 2011, 204 prostate cancer patients were treated per our institutional SBRT monotherapy protocol with a median follow-up of 4.8 years. They were ethnically diverse with a median age of 69 years (range, 48-90 years) (Table 1). The median prostate volume was $39 \mathrm{cc}$ and $8 \%$ of patients had prior procedures for benign prostatic hyperplasia (BPH). The median baseline IPSS was 8 and $28 \%$ of patients were using alpha-antagonists prior to SBRT. By D'Amico classification, $40 \%$ patients were low-, $52 \%$ intermediate-, and $8 \%$ high-risk. Fourteen percent of patients initiated ADT 3 months prior to the start of SBRT and for a mean duration of 5.5 months and a median duration of 3 months. Eighty-eight percent of patients were treated with $36.25 \mathrm{~Gy}$ in five $7.25 \mathrm{~Gy}$ fractions. The majority of patients had irritative voiding symptoms prior to treatment with a mean baseline IPSS-I score of 4.8 (Table 2; Figure 1A). At 1 month post-SBRT, the mean IPSS-I significantly increased to 6.5 ( $p<0.0001)$, but returned to baseline at 3 months $(p=0.7319)$ (Table 2; Figure 1A). This increase was of borderline clinical significance $(\mathrm{MID}=1.5)$. The median time to IPSS-I normalization was 3 months (Figure 2). The IPSS-I returned to baseline in $91 \%$ of patients by 6 months and $96 \%$ of patients by 2 years. At 2 years, the mean IPSS-I was close to baseline at $4.4(p<0.03)$. The mean IPSS-I significantly decreased from a baseline score of $4.8-3.7(p<0.0001)$ at 3 years post-SBRT. At 3 year post-SBRT, $59 \%$ of patients had an IPSS-I score that was less than their baseline. No baseline patient or treatment characteristics were significantly associated with improved irritative voiding symptoms on univariate or multivariate analysis 3 years following SBRT (data not shown). Alpha-antagonist utilization peaked

Table 2 | IPSS-irritative subscores.

\begin{tabular}{|c|c|c|c|c|c|c|c|c|c|c|}
\hline & Start & 1 & 3 & 6 & 9 & 12 & 18 & 24 & 30 & 36 \\
\hline \multicolumn{11}{|l|}{ (A) } \\
\hline Mean & 4.76 & 6.53 & 4.72 & 4.75 & 5.10 & 5.14 & 4.53 & 4.40 & 4.29 & 3.71 \\
\hline$p$-Value & & $<0.0001^{*}$ & 0.73 & 0.78 & 0.29 & 0.12 & 0.21 & $0.03 *$ & $0.003^{*}$ & $<0.0001^{*}$ \\
\hline STDEV & 3.08 & 3.25 & 2.86 & 2.91 & 3.24 & 3.35 & 3.23 & 3.31 & 3.10 & 3.40 \\
\hline MID & 1.54 & & & & & & & & & \\
\hline \multicolumn{11}{|l|}{ (B) } \\
\hline Mean & 2.69 & 5.49 & 3.40 & 3.64 & 3.85 & 4.11 & 3.55 & 3.20 & 3.07 & 2.48 \\
\hline$p$-Value & & $<0.0001^{*}$ & $0.0004^{*}$ & $0.0013^{*}$ & $0.0002 *$ & $<0.0001^{*}$ & $0.0042 *$ & $0.02 *$ & 0.23 & 0.43 \\
\hline STDEV & 1.42 & 2.78 & 2.04 & 2.23 & 2.51 & 3.08 & 2.45 & 2.40 & 2.15 & 2.22 \\
\hline MID & 0.71 & & & & & & & & & \\
\hline \multicolumn{11}{|l|}{ (C) } \\
\hline Mean & 6.80 & 7.56 & 6.01 & 5.81 & 6.29 & 6.12 & 5.52 & 5.49 & 5.41 & 4.91 \\
\hline$p$-Value & & $0.014^{*}$ & $0.001 *$ & $0.002 *$ & 0.106 & $0.027^{*}$ & $0.0002^{*}$ & $<0.0001^{*}$ & $<0.0001^{*}$ & $<0.0001^{*}$ \\
\hline STDEV & 2.91 & 3.37 & 2.95 & 3.09 & 3.41 & 3.32 & 3.61 & 3.66 & 3.42 & 3.90 \\
\hline MID & 1.45 & & & & & & & & & \\
\hline
\end{tabular}

(A) Mean IPSS-I patient-reported scores at baseline and during follow-up.

(B) Mean IPSS-I patient-reported scores with baseline IPSS score <8 for 102 patients.

(C) Mean IPSS-I patient-reported scores with baseline IPSS score $\geq 8$ for 102 patients. 

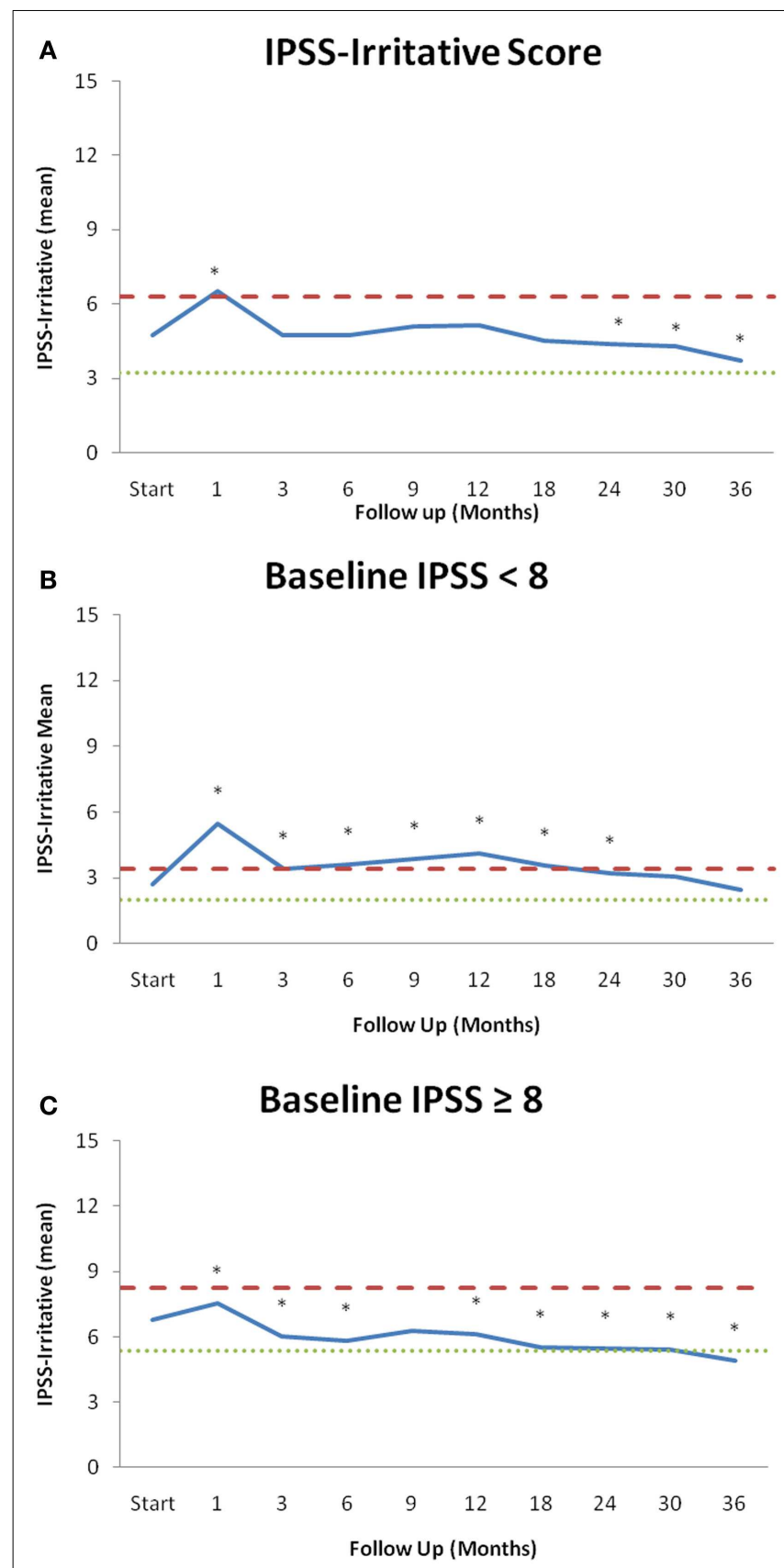

FIGURE 1 | IPSS-irritative subscores over the $\mathbf{3 6}$ months of follow-up stratified by baseline LUTS. (A) Mean IPSS-irritative score for all patients. (B) Mean values of patients with a mild baseline LUTS (IPSS $<8, n=102$ ). (C) Mean values of patients with a moderate to severe baseline LUTS (IPSS $\geq 8, n=102$ ). Changes in scores that are statistically significant different from baseline are marked with an asterisk $\left({ }^{*}\right)$. Thresholds for clinically significant changes in scores (1/2 standard deviation above and below the baseline) are marked with dashed lines. IPSS-irritative scores range from 0 to 15 with lower values representing a more favorable outcome.

1 month post-treatment, at $53 \%$ patient utilization, then slowly decreased to near baseline, with $32 \%$ of patients reporting use at 3 years (Figure $2 B$ ).
Individual irritative voiding symptoms (frequency, urgency, and nocturia) followed a similar trend (Table 3). At 1 month postSBRT, frequency significantly increased $(p<0.0001)$, but returned to baseline at 3 months ( $p=0.14$ ) (Table 3A). By 2 years, frequency had actually declined to below baseline $(p=0.002)$. Likewise, urgency increased significantly at 1 month and returned to baseline at 3 months $(p=0.64)$ (Table 3B). However, a second late protracted increase in urgency occurred between 9 and 18 months. Urgency returned to near baseline by 2 years post-SBRT. Similarly, nocturia increased transiently at 1 month and then again at 12 months (Table 3C; Figure 3). The pre-treatment and 3-year nocturia rates were similar.

Next, we assessed the impact of SBRT on irritative voiding symptoms 3 years following treatment on men with baseline mild (IPSS $<8,102$ men) LUTS versus moderate to severe (IPSS $\geq 8$, 102 men) LUTS (Tables 2B,C; Figures 1B,C) (32). In men with mild LUTS at baseline, the mean IPSS-I returned to near baseline by 3 years post-SBRT (Table 2B; Figure 1B). In men with moderate to severe LUTS at baseline, the mean IPSS-I significantly decreased from a baseline score of 6.8 to 4.9 at 3 years post-SBRT (Table 2C; Figure 1C). This decrease was both statistically $(p<0.0001)$ and clinically significant $(\mathrm{MID}=1.45)$.

At baseline, $63.1 \%$ of our cohort reported some level of bother due to urinary frequency with $17.7 \%$ of patients feeling it was a moderate to big problem (Table 4). At 1 month post-SBRT, moderate to big bother with urinary frequency increased to $28 \%$ $(p<0.0001)$, but reduced to $15.2 \%$ at 3 months $(p=0.7251)$. Although bother declined quickly, a second late transient increase in bother occurred at 12 months (Table 4). Despite this increase, only $14.6 \%$ of patients felt that weak urine stream and/or incomplete emptying was a moderate to big problem at 3 years post-SBRT $(p=0.2303)$.

\section{DISCUSSION}

Urinary toxicity following prostate radiotherapy involves both obstructive and irritative symptoms. Irritative voiding symptoms are more bothersome (4), yet they remain understudied. A better understanding of the pattern of irritative voiding symptoms following SBRT would enable clinicians to provide more realistic expectations to patients (33). In this study, we utilized validated QoL questionnaires to comprehensively evaluate irritative voiding symptoms following SBRT $(27,34)$.

An acute increase in irritative voiding symptoms occurs in most patients post-SBRT. It is believed to occur secondary to inflammation of the bladder neck/urethra. This study shows that SBRT acutely increases all irritative voiding symptoms (frequency, urgency and nocturia) in a similar manner. Furthermore, our results appear comparable to those reported for IMRT and brachytherapy (4). Irritative voiding symptoms may occur secondary to detrusor overactivity and could be treated as such (35). However, antimuscarinics are not routinely prescribed at our institution due to their known side effects and the potential risk of increased post-void residuals (36). Alternative approaches to managing acute irritative voiding symptoms post-SBRT should be explored.

Nocturia is the most bothersome LUTS (37). Nocturnal voiding as little as twice per night is associated with decreased quality 

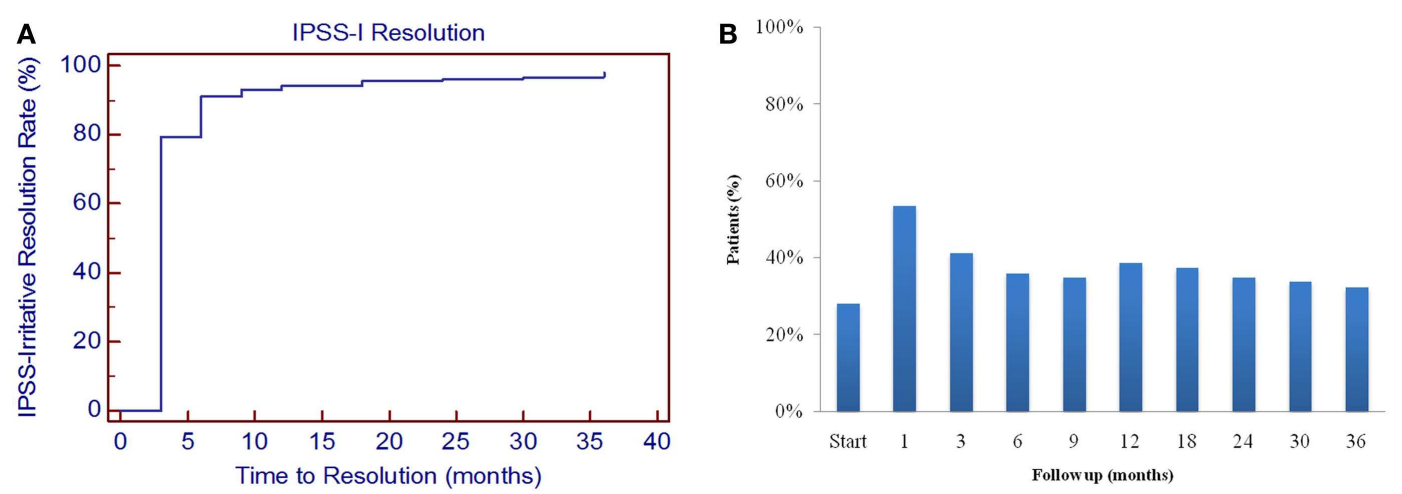

FIGURE 2 | Irritative voiding symptoms following SBRT for prostate cancer. (A) Time to IPSS-I resolution was determined by the number of months it took for the IPSS-I score to return to within one point of the baseline score. (B) Percent of patients utilizing alpha-antagonists at each time point.

Table 3 | Patient-reported responses to IPSS-irritative voiding questions at baseline and following SBRT for prostate cancer, recorded as the percent of the patient cohort.

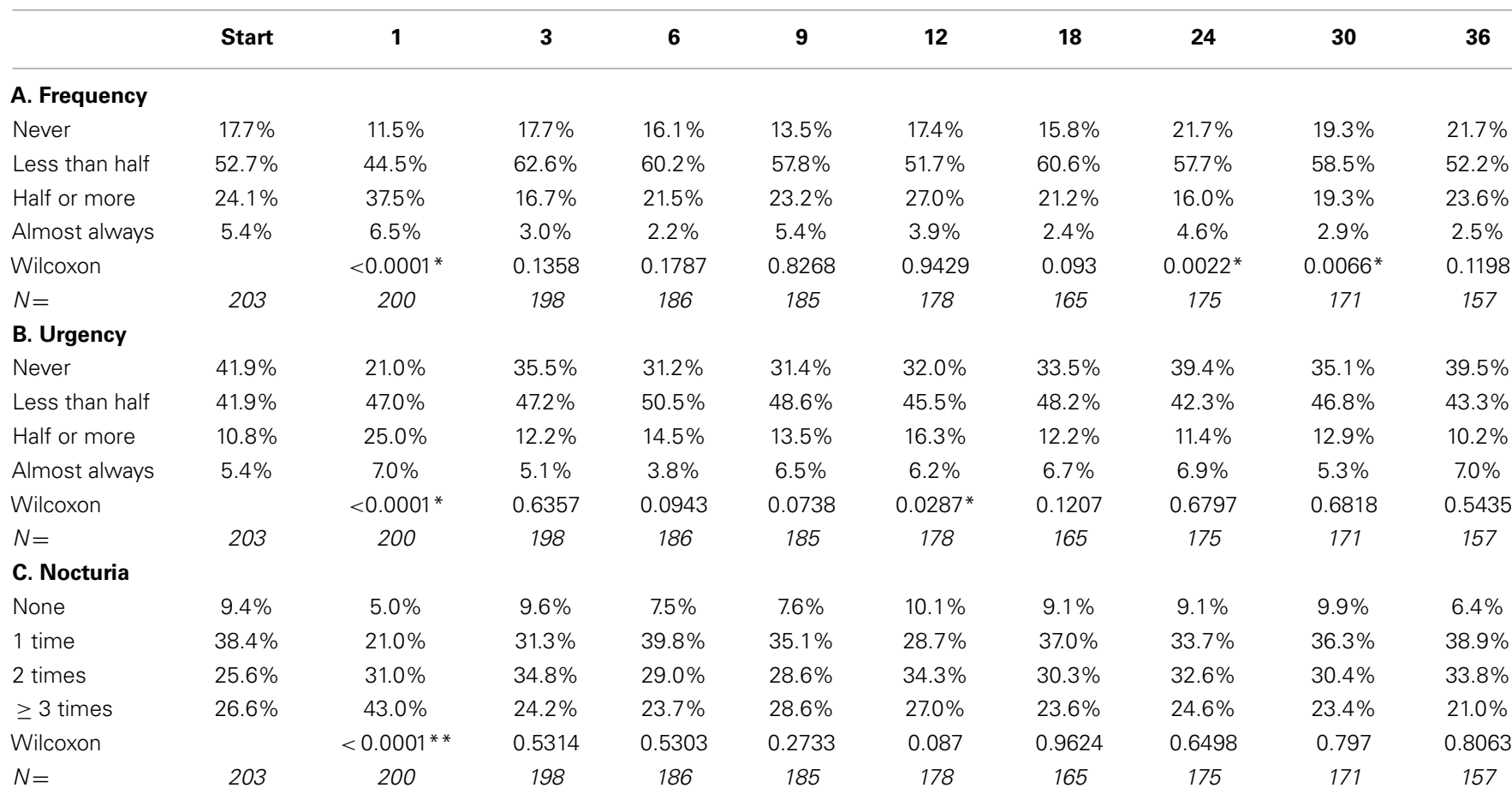

(A) Frequency (IPSS question 2) defined as how often a patient had to urinate within 2 hours of previously urinating.

(B) Urgency (IPSS question 4) defined as how often a patient found it difficult to postpone urination.

(C) Nocturia (IPSS question 7) defined as how often a patient needed to get up to urinate at night.

of life and may increase the risk of falls (11-13) To prevent falls and their associated morbidity, patients should be educated about their increased incidence following prostate radiation therapy. This study confirms the high incidence of baseline nocturia in elderly men with prostate cancer (38), while also showing a transient increase in nocturia at one month and twelve months post-treatment.

Due to its effectiveness and convenience, brachytherapy is a common treatment option for prostate cancer. Post-implant irritative voiding symptoms are a common toxicity that may impact long-term quality of life. IPSS resolution following brachytherapy varies from months to years $(29,39,40)$. Our mean IPSS-I scores returned to baseline within 3 months post-SBRT. A minority of patients experienced a transient increase in irritative voiding symptoms greater than six months after the completion of SBRT. As with brachytherapy, late urinary symptom flare (41-43) occurred in a minority of our patients and resolved with conservative management. Knowledge of this late transient increase 
Table 4 | Bother with frequency at baseline and following SBRT for prostate cancer (question 4e of the EPIC-26).

\begin{tabular}{|c|c|c|c|c|c|c|c|c|c|c|}
\hline & Start & 1 & 3 & 6 & 9 & 12 & 18 & 24 & 30 & 36 \\
\hline No problem & $36.9 \%$ & $17.5 \%$ & $35.9 \%$ & $34.9 \%$ & $32.4 \%$ & $31.5 \%$ & $39.4 \%$ & $42.3 \%$ & $39.8 \%$ & $42.0 \%$ \\
\hline Very small-small problem & $45.3 \%$ & $54.5 \%$ & $49.0 \%$ & $50.0 \%$ & $50.3 \%$ & $48.9 \%$ & $44.8 \%$ & $44.0 \%$ & $44.4 \%$ & $43.3 \%$ \\
\hline Wilcoxon & & $<0.0001 *$ & 0.7251 & 0.9337 & 0.4439 & 0.1663 & 0.6522 & 0.1859 & 0.5768 & 0.2303 \\
\hline$N=$ & 203 & 200 & 198 & 186 & 185 & 178 & 165 & 175 & 171 & 157 \\
\hline
\end{tabular}

Patients were stratified into three groups: no problem, very small to small problem, and moderate to big problem. The percentage of patients in each group at each time point is depicted in the chart over 3 years.

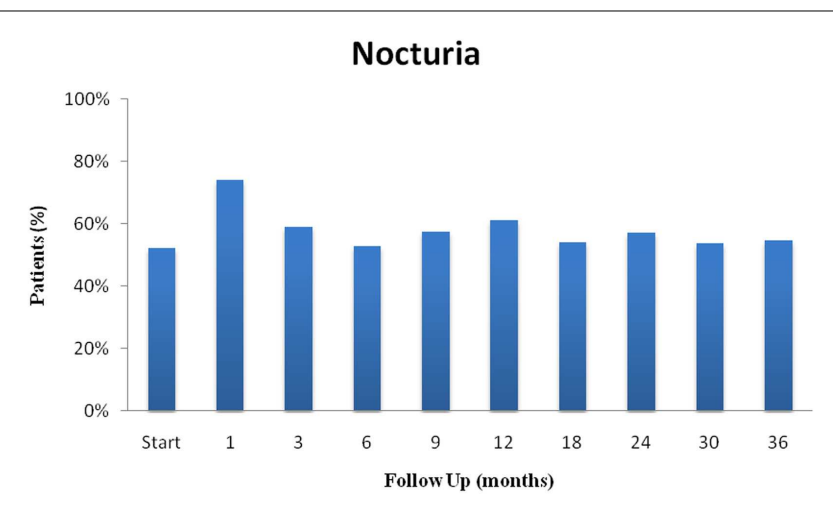

FIGURE 3 | Nocturia following SBRT for prostate cancer: (nocturia was defined as urinating $\geq \mathbf{2}$ times per night).

in irritative voiding symptoms will enable clinicians to address patient concerns.

Bother is defined as the degree of interference or annoyance caused by a symptom $(39,44)$. Similar to other radiation modalities, bother with urinary frequency plateaued at one month following treatment with $28 \%$ of men reporting it to be a moderate to big problem. This change compares favorably to the change reported at two months with conventionally fractionated EBRT (34\%) and brachytherapy (45\%) (4). As seen with EBRT, this increase in bother was transient and returned to baseline by 3 months post-SBRT. A second increase in bother occurred twelve months post-SBRT with $19.7 \%$ of patients reporting moderate to big bother at this time point. This change is comparable to that reported at twelve months with brachytherapy (20\%) (4). Unlike brachytherapy, bother following SBRT returned to near baseline by 2 years ( 7.1 vs. $20 \%$ ) and improved over baseline at 3 years.

Compared with prostatectomy, radiation therapy causes less incontinence at the expense of increased acute irritative voiding symptoms. However, this study shows that SBRT, like radical prostatectomy (45), may prevent age dependent increases in late irritative voiding symptoms. As seen following prostatectomy, men with moderate to severe LUTS benefited the most (45). Radical prostatectomy has been shown to improve irritative voiding symptoms within the first year following treatment $(4,46,47)$. It has been hypothesized that this is secondary to relief of prostatic obstruction. SBRT is ablative, with a decrease in prostate size seen within the first 3 years following treatment (22). Etiology of reduced irritative voiding symptoms 3 years post-SBRT is unclear, but may be due to prostate size reduction associated with SBRT treatment.

There were several limitations to this study. The EPIC-26 assesses bother associated with frequency but not bother related to urgency and nocturia (30). However, due to the high correlation between bother associated with these symptoms, it is unlikely that this would impact our conclusions. In addition, alternative mechanisms could explain the late improvement in irritative voiding symptoms such as prostate size reduction secondary to ADT and increased alpha antagonist usage (48). However, ADT usage was unlikely the main cause of improvement, as $<14 \%$ of patients received it with a median duration of only 3 months and a total of three patients, $1.5 \%$, receiving ADT for longer than 6 months. In addition, the baseline QOL assessment took place prior to treatment, on the first day of SBRT, 3 months after the initiation of ADT (49). Likewise, pre-treatment alpha antagonist usage was high and returned to baseline by $2-3$ years post-SBRT (50).

\section{CONCLUSION}

Stereotactic body radiation therapy treatment resulted in an acute increase in irritative voiding symptoms that peaked at one month post-treatment. These symptoms returned to baseline in the majority of patients by 3 months. Patients with moderate to severe LUTS can expect an improvement in their baseline irritative voiding symptoms years after treatment. Bother with urinary frequency was at baseline 2 years post-SBRT and improved by 3 years post-SBRT.

\section{ACKNOWLEDGMENTS}

This work was supported by the James and Theodore Pedas Family Foundation and NIH Grant P30CA051008.

\section{REFERENCES}

1. Wein AJ, Rovner ES. Definition and epidemiology of overactive bladder. Urology (2002) 60:7-12; discussion12. doi:10.1016/S0090-4295(02)01784-3

2. Milsom I, Stewart W, Thuroff J. The prevalence of overactive bladder. Am J Manag Care (2000) 6:S565-73.

3. Sexton CC, Coyne KS, Kopp ZS, Irwin DE, Milsom I, Aiyer LP, et al. The overlap of storage, voiding and postmicturition symptoms and implications for treatment seeking in the USA, UK and Sweden: EpiLUTS. BJU Int (2009) 103(Suppl 3):12-23. doi:10.1111/j.1464-410X.2009.08369.x

4. Sanda MG, Dunn RL, Michalski J, Sandler HM, Northouse L, Hembroff L, et al. Quality of life and satisfaction with outcome among prostate-cancer survivors. N Engl J Med (2008) 358:1250-61. doi:10.1056/NEJMoa074311

5. Abrams P, Kelleher CJ, Kerr LA, Rogers RG. Overactive bladder significantly affects quality of life. Am J Manag Care (2000) 6:S580-90. 
6. Bittner N, Merrick GS, Brammer S, Niehaus A, Wallner KE, Butler WM, et al. Role of trospium chloride in brachytherapy-related detrusor overactivity. Urology (2008) 71:460-4. doi:10.1016/j.urology.2007.10.023

7. Veenboer PW, Bosch JL. Long-term adherence to antimuscarinic therapy in everyday practice: a systematic review. J Urol (2014) 191:1003-8. doi:10.1016/j. juro.2013.10.046

8. Abrams P, Cardozo L, Fall M, Griffiths D, Rosier P, Ulmsten U, et al. The standardisation of terminology in lower urinary tract function: report from the standardisation sub-committee of the international continence society. Urology (2003) 61:37-49. doi:10.1016/S0090-4295(02)02243-4

9. Chapple CR, Artibani W, Cardozo LD, Castro-Diaz D, Craggs M, Haab F, et al. The role of urinary urgency and its measurement in the overactive bladder symptom syndrome: current concepts and future prospects. BJU Int (2005) 95:335-40. doi:10.1111/j.1464-410X.2005.05294.x

10. Tikkinen KA, Johnson TM II, Tammela TL, Sintonen H, Haukka J, Huhtala $\mathrm{H}$, et al. Nocturia frequency, bother, and quality of life: how often is too often? A population-based study in Finland. Eur Urol (2010) 57:488-96. doi:10.1016/j.eururo.2009.03.080

11. Kupelian V, Wei JT, O’Leary MP, Norgaard JP, Rosen RC, McKinlay JB. Nocturia and quality of life: results from the Boston area community health survey. Eur Urol (2012) 61:78-84. doi:10.1016/j.eururo.2011.05.065

12. Coyne KS, Zhou Z, Bhattacharyya SK, Thompson CL, Dhawan R, Versi E. The prevalence of nocturia and its effect on health-related quality of life and sleep in a community sample in the USA. BJU Int (2003) 92:948-54. doi:10.1111/j.1464-410X.2003.04527.x

13. Stewart RB, Moore MT, May FE, Marks RG, Hale WE. Nocturia: a risk factor for falls in the elderly. J Am Geriatr Soc (1992) 40:1217-20.

14. Parsons JK, Mougey J, Lambert L, Wilt TJ, Fink HA, Garzotto M, et al. Lower urinary tract symptoms increase the risk of falls in older men. BJU Int (2009) 104:63-8. doi:10.1111/j.1464-410X.2008.08317.x

15. McBride SM, Wong DS, Dombrowski JJ, Harkins B, Tapella P, Hanscom HN, et al. Hypofractionated stereotactic body radiotherapy in low-risk prostate adenocarcinoma: preliminary results of a multi-institutional phase 1 feasibility trial. Cancer (2012) 118:3681-90. doi:10.1002/cncr.26699

16. Freeman DE, King CR. Stereotactic body radiotherapy for low-risk prostate cancer: five-year outcomes. Radiat Oncol (2011) 6:3. doi:10.1186/1748-717X-6-3

17. Katz AJ, Santoro M, Diblasio F, Ashley R. Stereotactic body radiotherapy for localized prostate cancer: disease control and quality of life at 6 years. Radiat Oncol (2013) 8:118. doi:10.1186/1748-717X-8-118

18. King CR, Collins S, Fuller D, Wang PC, Kupelian P, Steinberg M, et al. Healthrelated quality of life after stereotactic body radiation therapy for localized prostate cancer: results from a multi-institutional consortium of prospective trials. Int J Radiat Oncol Biol Phys (2013) 87:939-45. doi:10.1016/j.ijrobp.2013. 08.019

19. King CR, Freeman D, Kaplan I, Fuller D, Bolzicco G, Collins S, et al. Stereotactic body radiotherapy for localized prostate cancer: pooled analysis from a multiinstitutional consortium of prospective phase II trials. Radiother Oncol (2013) 109:217-21. doi:10.1016/j.radonc.2013.08.030

20. Fowler JF. The radiobiology of prostate cancer including new aspects of fractionated radiotherapy. Acta Oncol (2005) 44:265-76. doi:10.1080/ 02841860410002824

21. Anwar M, Weinberg V, Chang AJ, Hsu IC, Roach M III, Gottschalk A. Hypofractionated SBRT versus conventionally fractionated EBRT for prostate cancer: comparison of PSA slope and nadir. Radiat Oncol (2014) 9:42. doi:10.1186/ 1748-717X-9-42

22. Low RN, Fuller DB, Muradyan N. Dynamic gadolinium-enhanced perfusion MRI of prostate cancer: assessment of response to hypofractionated robotic stereotactic body radiation therapy. AJR Am J Roentgenol (2011) 197:907-15. doi:10.2214/AJR.10.6356

23. Chen LN, Suy S, Uhm S, Oermann EK, Ju AW, Chen V, et al. Stereotactic body radiation therapy (SBRT) for clinically localized prostate cancer: the Georgetown University experience. Radiat Oncol (2013) 8:58. doi:10.1186/1748-717X-8-58

24. Lei S, Piel N, Oermann EK, Chen V, Ju AW, Dahal KN, et al. Six-dimensional correction of intra-fractional prostate motion with CyberKnife stereotactic body radiation therapy. Front Oncol (2011) 1:48. doi:10.3389/fonc.2011.00048

25. Vainshtein J, Abu-Isa E, Olson KB, Ray ME, Sandler HM, Normolle D, et al. Randomized phase II trial of urethral sparing intensity modulated radiation therapy in low-risk prostate cancer: implications for focal therapy. Radiat Oncol (2012) 7:82. doi:10.1186/1748-717X-7-82
26. Xie Y, Djajaputra D, King CR, Hossain S, Ma L, Xing L. Intrafractional motion of the prostate during hypofractionated radiotherapy. Int J Radiat Oncol Biol Phys (2008) 72:236-46. doi:10.1016/j.ijrobp.2008.04.051

27. Barry MJ, Fowler FJ Jr., O’Leary MP, Bruskewitz RC, Holtgrewe HL, Mebust WK, et al. The American urological association symptom index for benign prostatic hyperplasia. The measurement committee of the American urological association. J Urol (1992) 148:1549-1557; discussion1564.

28. Barry MJ, Williford WO, Fowler FJ Jr., Jones KM, Lepor H. Filling and voiding symptoms in the American urological association symptom index: the value of their distinction in a veterans affairs randomized trial of medical therapy in men with a clinical diagnosis of benign prostatic hyperplasia. J Urol (2000) 164:1559-64. doi:10.1097/00005392-200011000-00023

29. Merrick GS, Butler WM, Lief JH, Dorsey AT. Temporal resolution of urinary morbidity following prostate brachytherapy. Int J Radiat Oncol Biol Phys (2000) 47:121-8. doi:10.1016/S0360-3016(99)00525-8

30. Szymanski KM, Wei JT, Dunn RL, Sanda MG. Development and validation of an abbreviated version of the expanded prostate cancer index composite instrument for measuring health-related quality of life among prostate cancer survivors. Urology (2010) 76:1245-50. doi:10.1016/j.urology. 2010.01.027

31. Norman GR, Sloan JA, Wyrwich KW. Interpretation of changes in health-related quality of life: the remarkable universality of half a standard deviation. Med Care (2003) 41:582-92. doi:10.1097/00005650-200305000-00007

32. Lepor H, Kaci L. The impact of open radical retropubic prostatectomy on continence and lower urinary tract symptoms: a prospective assessment using validated self-administered outcome instruments. J Urol (2004) 171:1216-9. doi:10.1097/01.ju.0000113964.68020.a7

33. Symon Z, Daignault S, Symon R, Dunn RL, Sanda MG, Sandler HM. Measuring patients' expectations regarding health-related quality-of-life outcomes associated with prostate cancer surgery or radiotherapy. Urology (2006) 68:1224-9. doi:10.1016/j.urology.2006.08.1092

34. Wei JT, Dunn RL, Litwin MS, Sandler HM, Sanda MG. Development and validation of the expanded prostate cancer index composite (EPIC) for comprehensive assessment of health-related quality of life in men with prostate cancer. Urology (2000) 56:899-905. doi:10.1016/S0090-4295(00)00858-X

35. Blaivas JG, Weiss JP, Jones M. The pathophysiology of lower urinary tract symptoms after brachytherapy for prostate cancer. BJU Int (2006) 98:1233-1237; discussion1237. doi:10.1111/j.1464-410X.2006.06491.x

36. Hao N, Tian Y, Liu W, Wazir R, Wang J, Liu L, et al. Antimuscarinics and alphablockers or alpha-blockers monotherapy on lower urinary tract symptoms - a meta-analysis. Urology (2014) 83:556-62. doi:10.1016/j.urology.2013.10.034

37. DuBeau CE, Yalla SV, Resnick NM. Implications of the most bothersome prostatism symptom for clinical care and outcomes research. J Am Geriatr Soc (1995) 43:985-92.

38. De Langhe S, De Ruyck K, Ost P, Fonteyne V, Werbrouck J, De Meerleer G, et al. Acute radiation-induced nocturia in prostate cancer patients is associated with pretreatment symptoms, radical prostatectomy, and genetic markers in the TGFbetal gene. Int J Radiat Oncol Biol Phys (2013) 85:393-9. doi:10.1016/j.ijrobp.2012.02.061

39. Litwin MS, Gore JL, Kwan L, Brandeis JM, Lee SP, Withers HR, et al. Quality of life after surgery, external beam irradiation, or brachytherapy for early-stage prostate cancer. Cancer (2007) 109:2239-47. doi:10.1002/cncr.22676

40. Chan EK, Keyes M, Pickles T, Lapointe V, Spadinger I, McKenzie M, et al. Decline in acute urinary toxicity: a long-term study in 2011 patients with prostate brachytherapy within a provincial institution. Brachytherapy (2013) 13:46-52. doi:10.1016/j.brachy.2013.10.005

41. Cesaretti JA, Stone NN, Stock RG. Urinary symptom flare following I-125 prostate brachytherapy. Int J Radiat Oncol Biol Phys (2003) 56:1085-92. doi:10.1016/S0360-3016(03)00210-4

42. Crook J, Fleshner N, Roberts C, Pond G. Long-term urinary sequelae following 125iodine prostate brachytherapy. J Urol (2008) 179:141-145; discussion 146. doi:10.1016/j.juro.2007.08.136

43. Keyes M, Miller S, Moravan V, Pickles T, Liu M, Spadinger I, et al. Urinary symptom flare in $712125 \mathrm{I}$ prostate brachytherapy patients: long-term follow-up. Int J Radiat Oncol Biol Phys (2009) 75:649-55. doi:10.1016/j.ijrobp. 2008.11.043

44. Gore JL, Gollapudi K, Bergman J, Kwan L, Krupski TL, Litwin MS. Correlates of bother following treatment for clinically localized prostate cancer. J Urol (2010) 184:1309-15. doi:10.1016/j.juro.2010.06.012 
45. Prabhu V, Taksler GB, Sivarajan G, Laze J, Makarov DV, Lepor H. Radical prostatectomy improves and prevents age dependent progression of lower urinary tract symptoms. J Urol (2014) 191:412-7. doi:10.1016/j.juro.2013.08.010

46. Krupski T, Petroni GR, Bissonette EA, Theodorescu D. Quality-of-life comparison of radical prostatectomy and interstitial brachytherapy in the treatment of clinically localized prostate cancer. Urology (2000) 55:736-42. doi:10.1016/ S0090-4295(99)00597-X

47. Pardo Y, Guedea F, Aguilo F, Fernandez P, Macias V, Marino A, et al. Qualityof-life impact of primary treatments for localized prostate cancer in patients without hormonal treatment. J Clin Oncol (2010) 28:4687-96. doi:10.1200/JCO. 2009.25.3245

48. Malik R, Jani AB, Liauw SL. External beam radiotherapy for prostate cancer: urinary outcomes for men with high international prostate symptom scores (IPSS). Int J Radiat Oncol Biol Phys (2011) 80:1080-6. doi:10.1016/j.ijrobp.2010.03.040

49. Langenhuijsen JF, van Lin EN, Hoffmann AL, Spitters-Post I, Alfred Witjes $\mathrm{J}$, Kaanders $\mathrm{JH}$, et al. Neoadjuvant androgen deprivation for prostate volume reduction: the optimal duration in prostate cancer radiotherapy. Urol Oncol (2011) 29:52-7. doi:10.1016/j.urolonc.2009.03.024

50. Arscott WT, Chen LN, Wilson N, Bhagat A, Kim JS, Moures RA, et al. Obstructive voiding symptoms following stereotactic body radiation therapy for prostate cancer. Radiat Oncol (2014) 9:163. doi:10.1186/1748-717X-9-163
Conflict of Interest Statement: Sean P. Collins and Brian T. Collins serve as clinical consultants to Accuray Inc. The Department of Radiation Medicine at Georgetown University Hospital receives a grant from Accuray to support a research coordinator. The other authors declare that they have no competing interests.

Received: 05 September 2014; paper pending published: 21 September 2014; accepted: 07 October 2014; published online: 21 October 2014.

Citation: Rana Z, Cyr RA, Chen LN, Kim BS, Moures RA, Yung TM, Lei S, Collins BT, Suy S, Dritschilo A, Lynch JH and Collins SP (2014) Improved irritative voiding symptoms 3 years after stereotactic body radiation therapy for prostate cancer. Front. Oncol. 4:290. doi: 10.3389/fonc.2014.00290

This article was submitted to Radiation Oncology, a section of the journal Frontiers in Oncology.

Copyright (c) 2014 Rana, Cyr, Chen, Kim, Moures, Yung, Lei, Collins, Suy, Dritschilo, Lynch and Collins. This is an open-access article distributed under the terms of the Creative Commons Attribution License (CC BY). The use, distribution or reproduction in other forums is permitted, provided the original author(s) or licensor are credited and that the original publication in this journal is cited, in accordance with accepted academic practice. No use, distribution or reproduction is permitted which does not comply with these terms. 